\title{
New PARSEC database of alpha enhanced stellar evolutionary tracks and isochrones for Gaia
}

\author{
Xiaoting $\mathbf{F u}^{1}$, Alessandro Bressan ${ }^{1}$, Paola Marigo ${ }^{2}$, Léo Girardi ${ }^{3}$, \\ Josefina Montalban ${ }^{2}$, Yang Chen ${ }^{1,4}$, Ambra Nanni ${ }^{2}$ and \\ Antonio Lanza ${ }^{1}$ \\ ${ }^{1}$ SISSA - International School for Advanced Studies, \\ via Bonomea 265, 34136 Trieste, Italy \\ email: xtfu@sissa.it \\ ${ }^{2}$ Dipartimento di Fisica e Astronomia Galileo Galilei, Università di Padova, \\ Vicolo dell'Osservatorio 3, I-35122 Padova, Italy \\ ${ }^{3}$ INAF - Osservatorio Astronomico di Padova, \\ Vicolo dell'Osservatorio 5, I-35122 Padova, Italy \\ ${ }^{4}$ Department of Astronomy, University of Science and Technology of China,
} Hefei 230026, Anhui, China

\begin{abstract}
We present a new database of alpha enhanced evolutionary tracks and isochrones, computed with PARSEC (the PAdova \& TRieste Stellar Evolution Code). The new isochrones are tested against Color-Magnitude Diagrams of well studied Globular Clusters, tacking into account multiple population effects. They are also compared with observations of dwarf stars in the Solar vicinity. After these preliminary computations, we will provide the full sets of isochrones with chemical compositions suitable for Globular Clusters and Bulge stars, that will be fully implemented into galaxy simulators. We will also provide new models suitable for the analysis of unresolved stellar populations in early type galaxies.
\end{abstract}

Keywords. stars: evolution, stars: Hertzsprung-Russell diagram, stars: low-mass, stars: interiors, stars: abundances

\section{Range of parameters}

In current PARSEC isochrones (Bressan, et al. (2012), Chen, et al. (2014)), the initial metallicity is in the range from $\mathrm{Z}=0.0001$ to 0.07 (the present-day Suns metallicity is $\left.Z_{\odot}=0.01524\right)$. The initial mass is from $0.1 \mathrm{M}_{\odot}$ to $12 \mathrm{M}_{\odot}$. The alpha abundance $[\alpha / F e]=0$. The age of the star is from 0.1 Gyr to 14 Gyr. The RGB mass loss rate is $\eta=0.2$. Helium fraction $\mathrm{Y}$ follows the relation: $Y=0.2485+1.78 Z$. The new isochrones will be computed for low metallicity $(\mathrm{Z}=0.00001$ to 0.008$)$ stars and $\alpha$ enhancement $[\alpha / F e]=0.2,0.4$. The initial mass is from 0.1 to $350 \mathrm{M}_{\odot}$, and the stellar age from 0.1 Myr to 14 Gyr. Several values of Helium around the previous relation will be considered to allow multiple populations fitting with different helium. We recall here that when we change the fractional abundance by the number of heavy elements to obtain a new distribution, their fractional abundance by mass is re-normalized in such a way that the global metallicity, Z, is kept constant. 


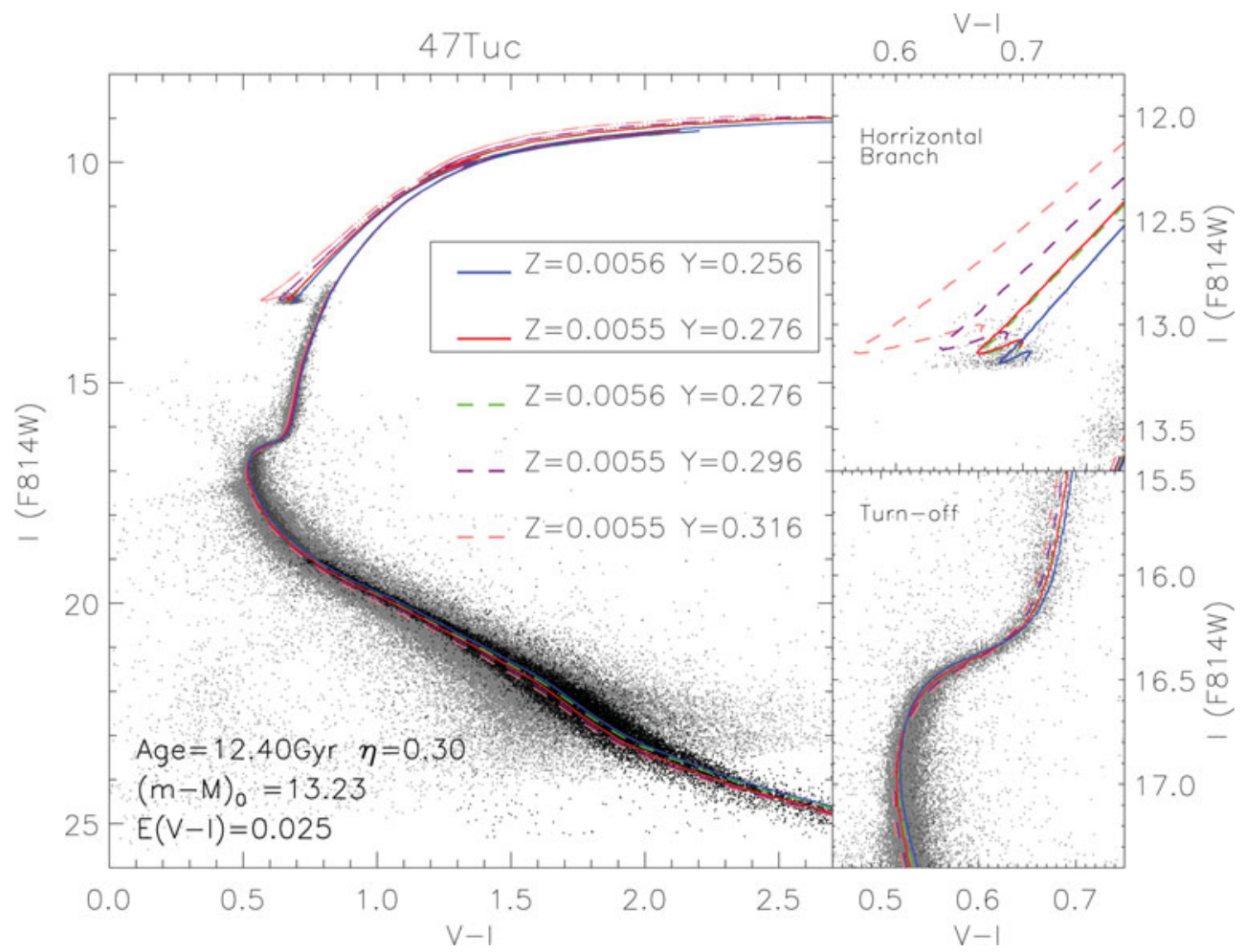

Figure 1. Isochrones fitting for 47 Tuc with alpha-enhanced metal mixture $\mathrm{Z}=0.0056 \mathrm{Y}=$ $0.256, \mathrm{Z}=0.0055 \mathrm{Y}=0.276$, and different helium contents around. Different lines represent different mixtures as the legend shows. The grey dot data are from the HST ACS Globular Cluster survey (Sarajedini, et al. (2007)), while the black dots are from Kalirai, et al. (2012). The isochrones are transformed from the theoretical tracks to the HST ACS/WFC color brand F606W (broad V) and F814W (I). The details of the Horizontal Branch region (the upper right panel) and Turn-off region (the lower right panel) are also shown.

\section{Preliminary computation}

We present here some results of the preliminary computations. The examples are with the metallicity of the Galactic Globular cluster 47Tuc (NGC104). The metal mixture with alpha enhancement for the two populations $(\mathrm{Z}=0.0056, \mathrm{Y}=0.256$ and $\mathrm{Z}=0.0055$, $\mathrm{Y}=0.276$ ) are based on recent spectroscopic and photometric surveys of 47Tuc stars in different evolutionary phases, from the main sequence close to the turn-off, to the giant phase (RGB, HB and AGB). In order to show how the variation of helium abundance Y affects the stellar evolutionary tracks and isochrones, and its connections with the RGB mass loss, some other $\mathrm{Y}$ values around the adopted ones for each population are also presented: $\mathrm{Z}=0.0056, \mathrm{Y}=0.276$ and $\mathrm{Z}=0.0055, \mathrm{Y}=0.296, \mathrm{Z}=0.0055, \mathrm{Y}=0.316$. Fig.1 shows the behaviors for different mixtures. Other isochrones with helium fraction between the selected values should locate between the plotted ones. With higher helium content, the horizontal branch is bluer, more extended and more luminous. There are two sets of data in the figure, the black ones are from Kalirai, et al. (2012), while the grey ones are the well-measured ACS GC survey data (Sarajedini, et al. (2007)) selected with method from Milone, et al. (2012)

The RGB mass loss rate $\eta$ also affects the horizontal branch evolution. Table 1 lists the average mass loss during RGB phase in the unit of $\mathrm{M}_{\odot}$ for different $\eta$. 
Table 1. The mass loss in the RGB, in unit of $\mathrm{M}_{\odot}$ for different RGB mass loss rate $\eta$.

\begin{tabular}{|l|l|l|l|}
\hline$\eta$ & $\mathbf{0 . 2 0} \mid \mathbf{0 . 2 5}$ & $\mathbf{0 . 3 0}|\mathbf{0 . 3 5}| \mathbf{0 . 4 0} \mid$ \\
\hline mass loss $\left(\mathbf{M}_{\odot}\right)|0.097| 0.123|0.149| 0.178 \mid$ & $0.208 \mid$ \\
\hline
\end{tabular}

\section{References}

Bressan, A., Marigo, P., Girardi, L., et al. 2012, MNRAS, 427, 127

Chen, Y., Girardi, L., Bressan, A., et al. 2014, MNRAS, 444, 2525

Kalirai, J., et al. 2012, AJ, 143, 11

Milone, J., et al. 2012, A\& $A, 540, \mathrm{~A} 16$

Sarajedini, A., et al. 2007, AJ, 133, 1658 